Authors

Sterling Backus, Matt Kirchner, Randy Lemons, David Schmidt, Charles Durfee, Margaret Murnane, and Henry Kapteyn 


\title{
Direct diode pumped Ti:sapphire ultrafast regenerative amplifier system
}

\author{
Sterling BACKus, ${ }^{1,2^{*}}$ MAtT KIRCHner, ${ }^{1}$ RANdy Lemons, ${ }^{4}$ DaVid \\ SChmidt, ${ }^{4}$ Charles Durfee, ${ }^{4}$ Margaret Murnane, ${ }^{1,3}$ AND Henry \\ KAPTEYN ${ }^{1,3}$ \\ ${ }^{1}$ Kapteyn-Murnane Laboratories Inc., 4775 Walnut Street \#102, Boulder, CO 80301, USA \\ ${ }^{2}$ Colorado State University, ECE, 1373 Campus Delivery, Fort Collins, CO 80523, USA \\ ${ }^{3}$ University of Colorado at Boulder, Department of Physics and JILA, Boulder, CO 80301, USA \\ ${ }^{4}$ Colorado School of Mines, Department of Physics, Golden, CO 80401, USA \\ *sbackus@kmlabs.com
}

\begin{abstract}
We report on a direct diode-pumped Ti:sapphire ultrafast regenerative amplifier laser system producing multi- $\mu \mathrm{J}$ energies with a repetition rate from 50 to $250 \mathrm{kHz}$. By combining cryogenic cooling of Ti:sapphire with high brightness fiber-coupled 450nm laser diodes, we for the first time demonstrate a power-scalable $\mathrm{CW}$-pumped architecture that can be directly applied to demanding ultrafast applications such as coherent high-harmonic EUV generation without any complex post-amplification pulse compression. Initial results promise a new era for Ti:sapphire amplifiers not only for ultrafast laser applications, but also for tunable $\mathrm{CW}$ sources. We discuss the unique challenges to implementation, as well as the solutions to these challenges.
\end{abstract}

(C)2017 Optical Society of America

OCIS codes: (320.0320) Ultrafast optics; (140.0140) Lasers and laser optics.

\section{References and links}

1. X. Zhang, E. Schneider, G. Taft, H. Kaptyen, M. Murnane, and S. Backus, "Multi-microjoule, MHz repetition rate Ti:sapphire ultrafast regenerative amplifier system," Opt. Express 20(7), 7015-7021 (2012).

2. S. Backus, in ASSP 2008 Proceedings (Japan, 2008).

3. T. B. Norris, "Femtosecond pulse amplification at $250 \mathrm{kHz}$ with a Ti:sapphire regenerative amplifier and application to continuum generation," Opt. Lett. 17(14), 1009-1011 (1992).

4. T. Imahoko, K. Takasago, M. Kamata, J. Sakuma, T. Sumiyoshi, H. Sekita, and M. Obara, "Development of a highly stable Yb: YAG thin disk pulsed green laser for high power Ti: sapphire based amplifier at $100 \mathrm{kHz}$ repetition rate," Appl. Phys. B 89(2-3), 217-222 (2007).

5. M. D. Young, S. Backus, C. Durfee, and J. Squier, "Multiphoton imaging with a direct-diode pumped femtosecond Ti:sapphire laser," J. Microsc. 249(2), 83-86 (2013).

6. T. Eidam, S. Hanf, E. Seise, T. V. Andersen, T. Gabler, C. Wirth, T. Schreiber, J. Limpert, and A. Tünnermann, "Femtosecond fiber CPA system emitting $830 \mathrm{~W}$ average output power," Opt. Lett. 35(2), 94-96 (2010).

7. S. Backus, J. Peatross, C. P. Huang, M. M. Murnane, and H. C. Kapteyn, "Ti:sapphire amplifier producing millijoule-level, 21-fs pulses at $1 \mathrm{kHz}$," Opt. Lett. 20(19), 2000-2002 (1995).

8. J. Limpert, F. Roser, D. N. Schimpf, E. Seise, T. Eidam, S. Hadrich, J. Rothhardt, C. J. Misas, and A. Tunnermann, "High Repetition Rate Gigawatt Peak Power Fiber Laser-Systems," IEEE J. Sel. Top. Quantum Electron 15(1), 159-169 (2009).

9. M. Nisoli, S. De Silvestri, and O. Svelto, "Generation of High-Energy $10 \mathrm{fs}$ pulses by a new pulse compression technique," Appl. Phys. Lett. 68(20), 2793-2795 (1996).

10. P. W. Roth, A. J. Maclean, D. Burns, and A. J. Kemp, "Directly diode-laser-pumped Ti:sapphire laser," Opt. Lett. 34(21), 3334-3336 (2009).

11. P. W. Roth, A. J. Maclean, D. Burns, and A. J. Kemp, Modelocking of a Diode-Laser-Pumped Ti:Sapphire Laser (IEEE, 2010).

12. C. G. Durfee, T. Storz, J. Garlick, S. Hill, J. A. Squier, M. Kirchner, G. Taft, K. Shea, H. Kapteyn, M. Murnane, and S. Backus, "Direct diode-pumped Kerr-lens mode-locked Ti:sapphire laser," Opt. Express 20(13), 1367713683 (2012).

13. K. Gürel, V. J. Wittwer, M. Hoffmann, C. J. Saraceno, S. Hakobyan, B. Resan, A. Rohrbacher, K. Weingarten, S. Schilt, and T. Südmeyer, "Green-diode-pumped femtosecond Ti:Sapphire laser with up to $450 \mathrm{~mW}$ average power," Opt. Express 23(23), 30043-30048 (2015).

14. A. Rohrbacher, V. Markovic, W. Pallmann, and B. Resan, in Solid State Lasers XXV: Technology and Devices, W. A. Clarkson and R. K. Shori, eds. (SPIE-Int Soc Optical Engineering, 2016), Vol. 9726. 
15. A. Müller, O. B. Jensen, A. Unterhuber, T. Le, A. Stingl, K. H. Hasler, B. Sumpf, G. Erbert, P. E. Andersen, and P. M. Petersen, "Frequency-doubled DBR-tapered diode laser for direct pumping of Ti:sapphire lasers generating sub-20 fs pulses," Opt. Express 19(13), 12156-12163 (2011).

16. S. Backus, C. G. Durfee Iii, G. Mourou, H. C. Kapteyn, and M. M. Murnane, "0.2-TW laser system at 1kHz," Opt. Lett. 22(16), 1256-1258 (1997).

17. P. F. Moulton, "Spectroscopic and laser characteristics of Ti: $\mathrm{Al}_{2} \mathrm{O}_{3}$," J. Opt. Soc. Am. B 3(1), 125-133 (1986).

18. I. L. B. G. V. Erbert, R. P. Hackel, and S. L. Jenkins, presented at the CLEO, 1991 (unpublished).

19. G. K. Samanta, S. C. Kumar, K. Devi, and M. Ebrahim-Zadeh, "High-power, continuous-wave Ti:sapphire laser pumped by fiber-laser green source at $532 \mathrm{~nm}$," Opt. Lasers Eng. 50(2), 215-219 (2012).

20. S. C. Kumar, G. K. Samanta, K. Devi, S. Sanguinetti, and M. Ebrahim-Zadeh, "Single-frequency, high-power, continuous-wave fiber-laser-pumped Ti:sapphire laser," Appl. Opt. 51(1), 15-20 (2012).

21. J. Dörring, A. Killi, U. Morgner, A. Lang, M. Lederer, and D. Kopf, "Period doubling and deterministic chaos in continuously pumped regenerative amplifiers," Opt. Express 12(8), 1759-1768 (2004).

22. C. E. Byvik and A. M. Buoncristiani, "Analysis of vibronic transitions in titanium doped sapphire using the temperature of the fluorescence spectra," IEEE J. Quantum Electron. 21(10), 1619-1624 (1985).

23. C. Ebert, T. Guiney, D. Irwin, and S. Patterson, in Laser Technology for Defense and Security XII, M. Dubinskii and S. G. Post, eds. (SPIE, 2016), Vol. 9834.

24. P. Moulton, Proceedings of the IEEE "Tunable Solid State Lasers," 80 (3), 348 (1992).

25. C. Hönninger, I. Johannsen, M. Moser, G. Zhang, A. Giesen, and U. Keller, "Diode-pumped thin-disk Yb:YAG regenerative amplifier," Appl. Phys. B 65(3), 423-426 (1997).

\section{Introduction}

Many scientific and industrial applications can benefit from the use of ultrashort sub-100 fs duration pulses with moderate energy but at $>100 \mathrm{kHz}$ repetition rates [1-4]. Typically, Ti:sapphire oscillator/amplifier systems have filled this role as a workhorse for research applications, with their unmatched ultrashort-pulse amplification performance and tunability [5]. More recently, Yb-based fiber lasers and other ultrafast sources have become morebroadly adopted for less-demanding applications, offering the advantages of a direct diodepumped architecture at the expense of pulse duration performance [6], while Ti:sapphire systems require complex intracavity-doubled green lasers for the pump source [7]. Nonlinear pulse compression or parametric amplification pumped by $\mathrm{Yb}$-based lasers can satisfy some needs for ultrafast pulses and tunability [8], but again increase complexity and reduce the reliability of the source, and often generate pulses with large pedestal and other poor characteristics [9]. Thus, the ideal ultrafast laser-diode pumped, simple, reliable, and with uncompromising performance-remains to be realized.

Recently, however, direct diode pumping of Ti:sapphire itself has become feasible [10 14]. GaN and other short-wavelength laser diodes have become available, driven in large part by the potential for driving phosphors for advanced white-light applications such as projectors and laser-driven headlights. These diodes can provides several watts of power in a single emitter, with $450 \mathrm{~nm}$ and more-recently $520 \mathrm{~nm}$ diodes ( $\mathrm{InGaN}$ ) proven capable of driving laser action in Ti:sapphire. This has led to the use of single-emitter diodes, or a small number of multiplexed single emitters, to power $\mathrm{CW}$, saturable absorber modelocked, and finally Kerr-lens modelocked Ti:sapphire lasers - the latter with the ability to generate pulses of 1020 fs duration [12]. In addition, doubled diode lasers have also been used in oscillator demonstrations [15].

The next frontier in direct-diode-pumped Ti:sapphire (DDPTS) is to scale-up to highpower ultrafast laser amplifier systems. The potential for success, however, is not obvious because of a number of challenges. Scaling to high power requires maintaining sufficient brightness in the pump source, while allowing for a scalable architecture. The gain in a "good" laser material depends primarily on the peak gain cross section and the lifetime of the gain medium as $G=\mathrm{e}^{\Delta \mathrm{N} \sigma_{\mathrm{g}} L}=\mathrm{e}^{(\mathrm{P} / \mathrm{hv}) \tau \sigma_{\mathrm{g}} L}$ where $\mathrm{P}$ is the absorbed pump power, $\tau$ is the lifetime, $\Delta \mathrm{N}$ is the inversion density, hv is the lasing photon energy, $\mathrm{L}$ is the crystal length, and $\sigma_{\mathrm{g}}$ is the gain cross section. In comparison with diode-pumped $\mathrm{Yb}$ laser systems (for example, Yb:KGW $\sigma_{\mathrm{g}}=3 \times 10^{-20} \mathrm{~cm}^{2} ; \tau=600 \mu \mathrm{sec}$ ) the power density required for similar gain in Ti:sapphire $\left(\sigma_{\mathrm{g}}=3 \times 10^{-19} \mathrm{~cm}^{2}, \tau=3.9 \mu \mathrm{sec}\right.$ at low temperature $)$ is approximately $15 \mathrm{x}$ 
higher. Furthermore, Yb materials can be quite heavily-doped (up to 25\%), making it possible to focus the pump light tightly and absorb it in a relatively short length in the crystal, before the focal spot diverges. By contrast, doping of $\mathrm{Ti}^{3+}$ in sapphire is limited to $\sim 0.25-0.41 \%$ by weight. At higher doping levels, optical quality is degraded and excited-state absorption (ESA) increases loss. In contrast to the very high power $>100 \mathrm{~W}$ diode bars available in the IR, multi-emitter diode bars at $450 \mathrm{~nm}$ are not available. Since only discrete-element blue laser diodes are available, multiplexing many diodes into a multimode fiber is the only current practical high-power diode-pumping technique potentially adaptable for Ti:sapphire pumping. However, fiber coupling also comes at the expense of overall brightness. The challenge thus is how to maximize the pump brightness delivered to the material, to allow for the focused pump light to be absorbed within a Ti:sapphire crystal with length comparable to the confocal parameter of the pump laser focus. An additional challenge is that ultrashortpulse regenerative amplifier - the most interesting candidate for scaling-up in powernecessarily includes lossy elements (Pockels cell, polarizing beamsplitters) requiring an $\sim 10 \%$ gain in the laser medium to exceed threshold. The prospects for success in powerscaling to DDPTS are thus not obvious.

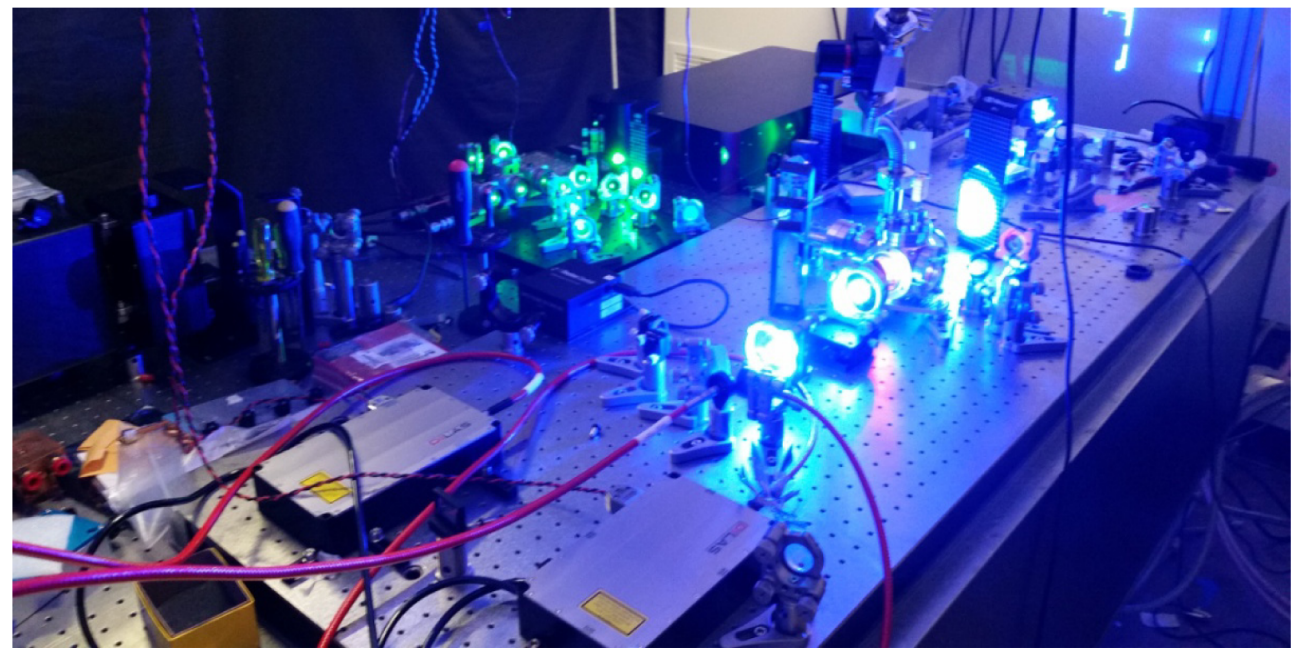

Fig. 1. Fully diode-pumped ultrafast Ti:sapphire oscillator amplifier system. The Kerr-lensmodelocked Ti:sapphire oscillator is pumped with 520nm diodes $(1.7 \mathrm{~W})$, while the amplifier is pumped by high-power fiber-coupled $450 \mathrm{~nm}$ diodes (up to 100W) and is cryogenically cooled.

Nevertheless, these obstacles can be overcome. In this work we present what we believe is the first direct diode-pumped Ti:sapphire oscillator/amplifier system. By using high-power spatially and polarization-multiplexed single-emitter $450 \mathrm{~nm}$ laser diodes coupled into a multimode fiber as the pump source, we demonstrate both $\mathrm{CW}$ operation of a Ti:sapphire laser with output power up to $11 \mathrm{~W}$, as well as pulsed operation at up to $250 \mathrm{kHz}$ with $\sim 1 \mathrm{~W}$ output. This system was also seeded by a DDPTS Kerr-lens modelocked laser pumped by 520 nm diodes.

We believe that this is the also the first demonstration of pumping a Ti:sapphire laser or amplifier with a fiber-coupled source of any type - a substantial advantage for engineering of stable and reliable systems (IR fiber based systems use a free space second harmonic generator at the exit). We find also that there are qualitative differences in the character of pumping of Ti:sapphire by $450 \mathrm{~nm}$ compared with $520-540 \mathrm{~nm}$, that make the use of cryogenic cooling for the Ti:sapphire crystal especially advantageous and possibly necessary [16]. By demonstrating the basic feasibility of this laser architecture, we expect further refinements can reduce the lasing threshold and result in further rapid increases in overall 
efficiency. In particular, although high power $532 \mathrm{~nm}$ lasers are available as pulsed sources (1-200 kHz, up to $500 \mathrm{~W})$, the CW pump power from these $450 \mathrm{~nm}$ modules is an order of magnitude less expensive than $\mathrm{CW}$ frequency-doubled alternatives (and likely to further come-down in cost), making very high rep-rate, high-power systems running at $100 \mathrm{kHz}$ to $>$ $\mathrm{MHz}$ feasible for the first time.

\section{Laser system}

Figure 1 shows a photo of the laser oscillator/amplifier in operation. A modelocked Ti:sapphire oscillator is pumped by green $520 \mathrm{~nm}$ laser diodes, while the amplifier is pumped by $2 \mathrm{x}$ high-power $450 \mathrm{~nm}$ fiber-coupled diode arrays. Other than being diode-pumped by multiplexed $520 \mathrm{~nm}$ diodes with excellent $\mathrm{M}^{2} \sim 1$ in the fast axis and $\mathrm{M}^{2} \sim 3$ in the slow axis, [Patent US8976821] the laser is a standard Kerr-lens modelocked Ti:sapphire laser. The 35 nm FWHM bandwidth shown in Fig. 2(blue) is sufficient for a $20 \mathrm{fs}$ pulse.

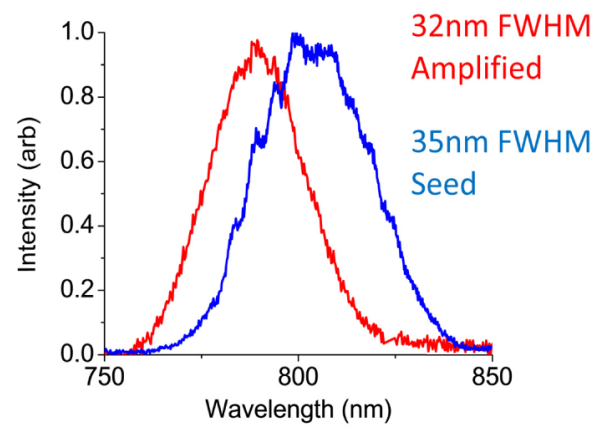

Fig. 2. (blue) input spectrum from direct diode pumped oscillator. (red) Output spectrum from regenerative amplifier system.

The amplifier cavity in Fig. 3 is designed to mode-match relay-imaged output of the $450 \mathrm{~nm}$ diode module (DILAS GmbH). This custom fiber-coupled array emits $50 \mathrm{~W}$ from a $200 \mu \mathrm{m}$ core multimode fiber with a $0.22 \mathrm{NA}$ at the output. The pump beam is imaged 1:1 into a $0.25 \%$ doped, normal incidence AR coated $8 \mathrm{~mm}$ long Ti:sapphire crystal (GTAT/Crystal Systems, FOM $>300$ ) using $\mathrm{f}=100 \mathrm{~mm}$ AR coated achromats. Because of the high divergence of the pump beam, it was focused (to $200 \mu \mathrm{m}$ ) near the entrance face of the crystal for maximum gain. The $\sim 15 \times 25 \mathrm{~cm}$ diode packages are seen in the lower left of the picture in Fig. 1.

However, prior to implementing the regenerative amplifier, we attempted $\mathrm{CW}$ lasing by removing the regenerative amplifier components (polarizer, Pockels cell) and operating with all high-reflectors to minimize loss. Laser action with the crystal held at room temperature was not obtained. This could be for two reasons. First, the thermal lensing at a pump power $>20 \mathrm{~W}$ (lasing threshold in cryogenic operation) is predicted to be severe at $<\sim 1 \mathrm{~cm}$. 


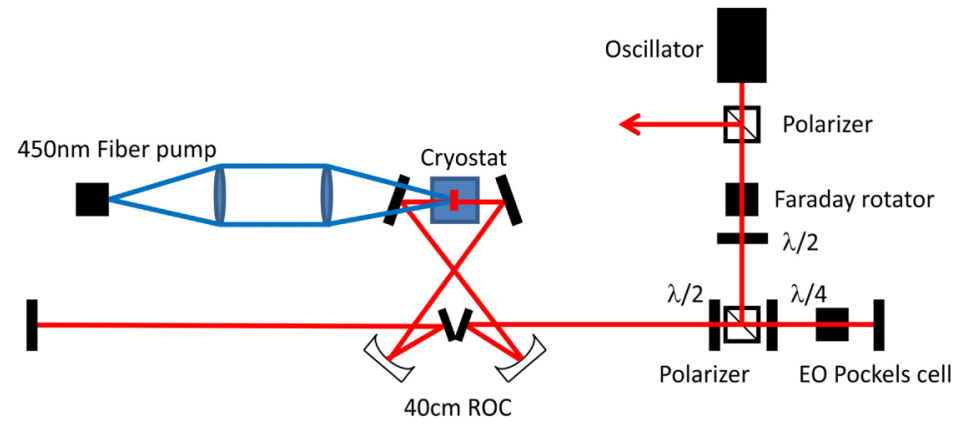

(a)

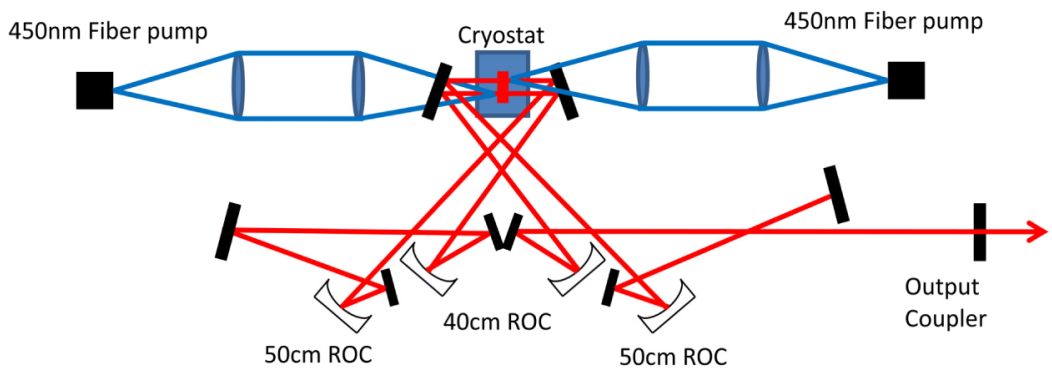

(b)

Fig. 3. (a) Regenerative amplifier setup for demonstration of direct diode-pumped Ti:sapphire amplifier system. (b) Double focus CW cavity for high power 100W tests.

Second, we observed that the fluorescence yield for the crystal when pumped at $450 \mathrm{~nm}$ was noticeably low; i.e. fluorescence at $\sim 800 \mathrm{~nm}$ clearly visible when the crystal was cooled, all-but disappeared in room-temperature pumping. This effect is discussed later in this paper, but low fluorescence also is consistent with low gain. All subsequent demonstrations were done with a $130 \mathrm{~K}, 75 \mathrm{~W}$ capacity cryocooler (Cryospectra $\mathrm{GmbH}$ ). Ti:sapphire is known to have a polarization-dependent absorption cross section [17]. However, this effect is reduced at $450 \mathrm{~nm}$. The ratio of absorption cross-sections for the $\|$ and $\perp$ axes is 1.76 at $450 \mathrm{~nm}$, and 2.42 at $532 \mathrm{~nm}$. Therefore using $450 \mathrm{~nm}$ light makes it more practical to use an un-polarized pump. The absorption of the $450 \mathrm{~nm}$ pump light was measured at $\sim 70 \%$.

Figure 4(a) shows output as a function of pump power from the laser cavity under CW operation using a single $50 \mathrm{~W}$ pump module and $5 \%$ output coupler. We observe a threshold of $21 \mathrm{~W}$, a $24 \%$ slope efficiency and up to $3 \mathrm{~W}$ of average power. This corresponds to approximately $8.6 \%$ overall efficiency in converting absorbed pump light to output power; the observed slope efficiency is already competitive with $\mathrm{CW}$ green-pumped Ti:sapphire lasers. Although the threshold for lasing is considerably higher than any known past work due to the rather large pump laser mode and the current overall conversion efficiency is less than the $25 \%$ that can be achieved with green pumping, we note that the diode-pumped system is almost $14 \mathrm{x}$ less expensive: the highest power solid-state green laser that we are aware of is $18 \mathrm{~W}$ for approximately $150 \mathrm{k} \$(\$ 8300 / \mathrm{W})$, while our current system was $100 \mathrm{~W}$ for approximately $60 \mathrm{k} \$(\$ 600 / \mathrm{W})$. We calculated the gain distribution in the Ti:sapphire crystal by assuming the fiber output was imaged with unity magnification and had an $\mathrm{M}^{2}$ value of 150 , consistent with the 0.22 numerical aperture of the fiber. This simple model predicts a single-pass gain of $6.3 \%$ at $21 \mathrm{~W}$ absorbed. If we assume $0.25 \%$ and $0.1 \%$ loss on antireflective and high reflective coatings, respectively, and a figure of merit of 400 for the 
crystal, the net round trip loss would be $10 \%$. Therefore the calculated gain is slightly higher than required to reach threshold. This could be accounted for by about $2.5 \%$ additional cavity loss, or a slightly more diffuse pump mode. However, the predicted slope efficiency of $10.6 \%$ is considerably less than observed. We will return to a discussion of anomalous behavior below.

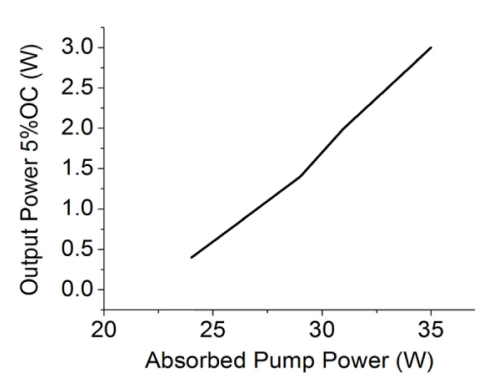

(a)

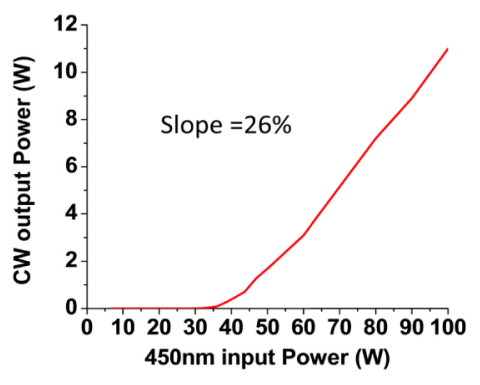

(b)

Fig. 4. CW operation of the Ti:sapphire cavity pumped by fiber-coupled $450 \mathrm{~nm}$ modules. (a) Laser slope efficiency plot for the cavity of Fig. 3(a), sans PC and polarizers and using a single $50 \mathrm{~W}$ pump module. Threshold is at $21 \mathrm{~W}$ of pump, and slope efficiency is $24 \%$. (b) Slope efficiency curve for a CW configuration using 2x 50W pump modules Fig. 3(b). In this case, the two pump modules are focused from opposite sides of the crystal into two separate spots, and the laser cavity passes through both spots. This configuration shows a similar threshold, but allows for operation considerably farther above threshold. The maximum output power of $11 \mathrm{~W}$ exceeds that of any other published work on $\mathrm{CW}$ Ti:sapphire, due to the use of very high $\mathrm{CW}$ pump power.

To make use of both pump modules, a double-focus cavity design was implemented, with the pump modules incident from opposite sides but focused on separate spots next to each other in the same crystal shown in Fig. 3(b). This configuration shows a similar threshold and slope efficiency $(\sim 26 \%)$, but allows for operation considerably farther above threshold. The maximum output power of $11 \mathrm{~W}$ exceeds that of any known published work on CW solid-state pumped Ti:sapphire, due to the use of very high CW pump power. In past work, a set of 6 Arion lasers with a cumulative pump power of $103 \mathrm{~W}$ focused in into three cryogenically-cooled crystals generated $43 \mathrm{~W}$ output [18], and doubled fiber laser pumped Ti:sapphire systems have generated up to $2.7 \mathrm{~W}[19,20]$. In contrast, the pump modules used in this work only requires low flow water cooling; unlike the case of fiber laser pumping, no post-fiber external frequency-doubling is required.

The next step was to set up the cavity for q-switching by inserting the polarizer, half- and quarter-waveplates, and Pockels cell into the cavity, and replacing the OC with a high reflectivity mirror. The cavity was $\mathrm{q}$-switched, producing $\sim 10 \mathrm{~ns}$ narrowband pulses at $250 \mathrm{kHz}$. Threshold for operation was similar to $\mathrm{CW}$ lasing at just over $22 \mathrm{~W}$ of absorbed $450 \mathrm{~nm}$ pump light. The cavity only operated with a crystal temperature below $133 \mathrm{~K}$, owing to strong thermal lensing above that temperature.

Pulses from the Ti:sapphire oscillator $(0.6 \mathrm{~nJ})$ were then injected into the regenerative cavity with no stretcher, using one 50W pump module. The pulses circulated in the cavity for a buildup time of 2.6us. Subtracting the oscillator average power from the output power gives an output energy of $3.8 \mathrm{uJ}$, for a total gain of $38 \mathrm{~dB} @ 250 \mathrm{kHz}$. From the buildup characteristics, the unsaturated gain per pass was estimated at $6 \%$. Including a $5 \%$ loss per pass sets the estimated gain per pass at approximately $11 \%$. 


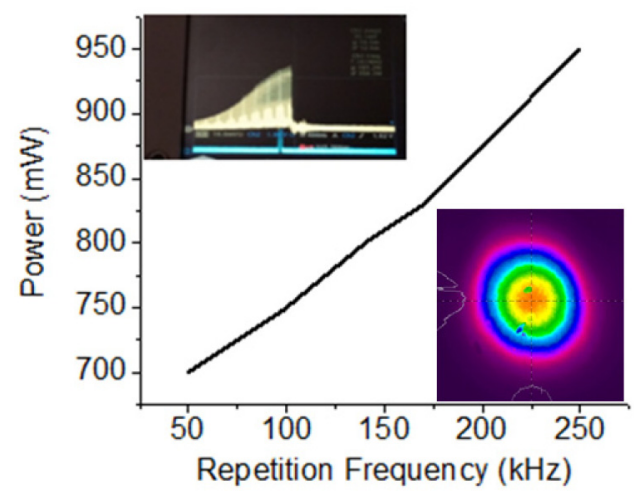

Fig. 5. Demonstration of direct diode-pumped ultrafast Ti:sapphire laser-amplifier. Graph shows power vs repetition-rate for a preliminary cavity optimization, pumped by $45 \mathrm{~W}$. Inset (upper left) shows regenerative amplifier cavity buildup, and inset (lower right) shows beam image.

Figure 5 shows the output power (minus oscillator background) as a function of output repetition rate. Inset is an oscilloscope capture of the pulse build up inside the regenerative cavity. When the oscillator input energy was cut in half, bifurcation [21] in the output repetition frequency due to amplifier under-seeding was observed, where the energy of the output pulses alternates in magnitude. Figure 2(red) shows the output spectrum of the amplifier at $250 \mathrm{kHz}$, with a TL of $\sim 30 \mathrm{fs}$. The gain narrowing of the spectrum was expected to be quite a bit more severe than is observed, which merits further investigation. We checked carefully for whether any of the output is ASE, but see no evidence in the photodiode trace which can often be observed as a DC offset, further follow-up is underway. In addition, ongoing studies are underway for the double focus cavity to utilize as a regenerative amplifier, with a goal of $>5 \mathrm{~W}$ output.

Starting with the modeled spatially-dependent pump absorption described earlier, we calculated the amplifier gain dynamics. To allow the energy extraction to equilibrate with the $\mathrm{CW}$ pumping, we ran the model for several injection cycles. With the same gain distribution as used in the $\mathrm{CW}$ laser model, 5\% per pass loss, the model predicts an energy output of approximately $3 \mathrm{uJ}$ and a build-up time of $2.4 \mathrm{us}$. As in the measurements, we observe that the energy output alternates between low and high energies when the amplifier is under-seeded.

\section{Fluorescence behavior}

In implementation of this laser, we made some observations of some unanticipated behavior. The photos of Fig. 6 show the fluorescence emerging from the crystal in the cryostat at two temperatures at constant pump power.

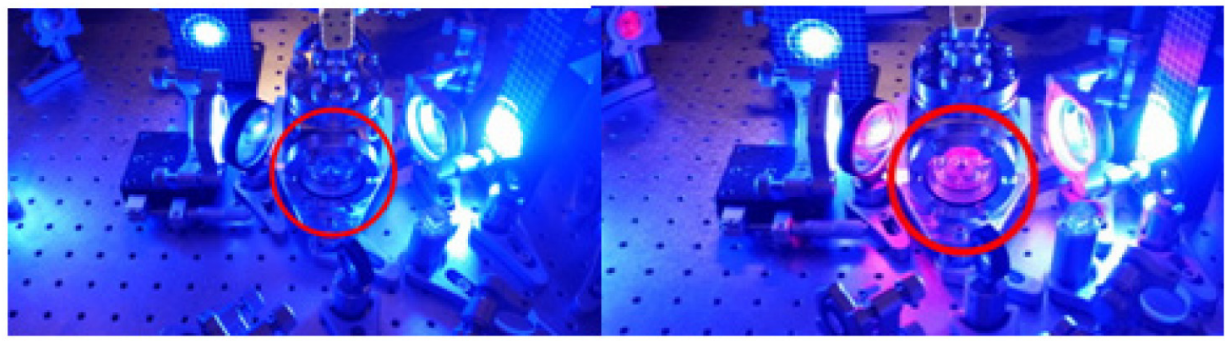

Fig. 6. Marked quenching of fluorescence from Ti:sapphire pumped with with high-power 450 $\mathrm{nm}$ diodes. Left: $50 \mathrm{~W}$ input power, Ti:sapphire crystal at $295 \mathrm{~K}$. Note that the cryocell window is dark. Right: same, but crystal at $93 \mathrm{~K}$. Note that this behavior is not as marked with $532 \mathrm{~nm}$ pumping, suggesting new gain dynamics in the system. 
With the crystal at room temperature in Fig. 5(a), fluorescence visible to the eye (or camera) is barely visible, but increases markedly at low temperature, in the case of $93 \mathrm{~K}$, Fig. 5 (b). Although increased fluorescence at low temperature is noticeable with green-pumped cryocooled Ti:sapphire, for $450 \mathrm{~nm}$ this is much more dramatic. To probe this behavior further, we measured the fluorescence intensity as a function of temperature with $450 \mathrm{~nm}$ and $532 \mathrm{~nm}$ pumping, adjusted for the same $10 \mathrm{~W}$ power absorbed in the crystal (which was not observed to change appreciably with temperature).

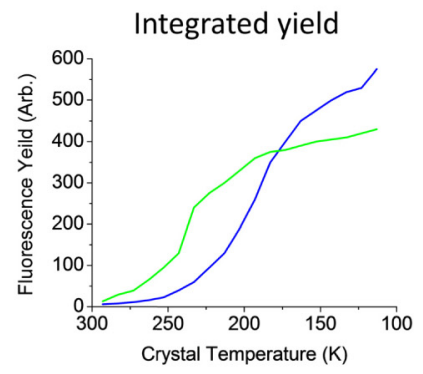

(a)

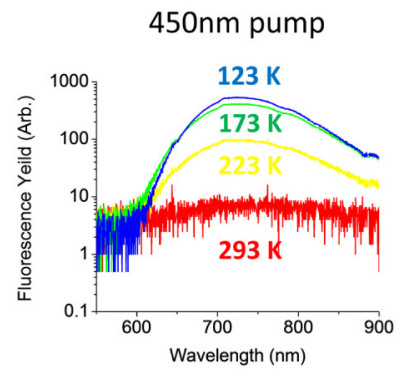

(b)

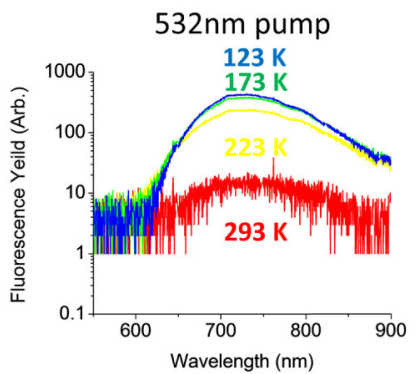

(c)

Fig. 7. (a) Integrated fluorescence yield vs temperature for conventional Ti:sapphire pumping and (green) pumping with 450nm diodes (blue), showing an advantage using 450nm diodes at cryogenic temperatures below $188 \mathrm{~K}$. Integrated spectral power as a function of temperature showing a distinct difference in output fluorescence between the two pump wavelengths. The $450 \mathrm{~nm}$ pump is un-polarized, where the $532 \mathrm{~nm}$ pump is polarized along the c-axis. (b) Raw data showing spectral fluorescence content for select temperatures pumping with 450nm laser. (c) Raw data showing spectral fluorescence content for select temperatures pumping with $532 \mathrm{~nm}$ laser.

Figure 7(a) shows the spectrally-integrated fluorescence yield for both cases. We do not see any marked difference in the shape of the fluorescence spectrum between 450 and $532 \mathrm{~nm}$ pumping, or changes with temperature. At $\sim 188 \mathrm{~K}$, the $450 \mathrm{~nm}$ pumped fluorescence exceeds that from the $532 \mathrm{~nm}$ pumped crystal, and continues to rise as the temperature decreases. Note that these data were normalized for absorbed pump power — not absorbed photons - and thus if the fluorescence yield in both cases rises to $\sim 1$ as nonradiative decay channels shut-off below $150 \mathrm{~K}$ as has been interpreted in past work by Byvik and Buonchristiani [22] (and is consistent with the green line of Fig. 7), the $450 \mathrm{~nm}$ curve should level-out $\sim 15 \%$ lower than $532 \mathrm{~nm}$, reflecting the larger quantum defect for pumping. Instead, the character of the temperature dependence is quite different. If the fluorescence yield for $532 \mathrm{~nm}$ pumping at low temperature is $\sim 1$, this would mean that the fluorescence yield is $>1$ for $450 \mathrm{~nm}$ pumping. There are other differences such as the pump polarization, so that more careful investigation is necessary. However, a yield of $>1$ may be possible due to a carrier multiplication effect, as has been studied extensively for possible photovoltaic applications and is also observed for $793 \mathrm{~nm}$ pumping of Tm:fiber [23]. It is known that the relaxation dynamics in Ti:sapphire creates a gain bottleneck for pulses in the $\sim 10 \mathrm{ps}$ range, so that a hot ground-state excitation distribution could result in absorption of $450 \mathrm{~nm}$ light into states high enough in the excited state band to relax to the bottom of the excited state level through an Auger-type process [24] (this process would actually cool the crystal as-well). If this interpretation is correct, pumping with even shorter wavelengths (i.e. $\sim 400 \mathrm{~nm} \rightarrow \mathrm{h} v=2$ xpeak of fluorescence) may further enhance the effect, and allow for very efficient ultrashort-pulse amplification in a material previously thought to have a large quantum defect. Although this premise remains to be conclusively proven, it is also bolstered by the fact that observed slope efficiency are higher than might otherwise be expected for a rather less-than-optimal pumping geometry. Showing that pumping with higher-energy photons actually improves the efficiency would be a surprising and technologically important result. 


\section{Conclusion and summary}

In conclusion, in this work we present what we believe to be the first demonstrations of a Ti:sapphire laser and regenerative amplifier driven by fiber-coupled direct diode pumping. We have to date achieved $11 \mathrm{~W}$ of $\mathrm{CW}$ power, and demonstrated regenerative amplification of ultrafast pulses. The output powers achieved both in $\mathrm{CW}$ and amplifier operation are primarily limited by the pump brightness. The use of fiber-coupling of the pump light allows for straightforward scaling to higher pump powers, as the performance of these modules currently is rapidly improving, with increased power output and decreased fiber diameter. Furthermore, the output power of single emitters continues to improve with output power at $450 \mathrm{~nm}, 465 \mathrm{~nm}$ and $520 \mathrm{~nm}$ of $6 \mathrm{~W}, 4 \mathrm{~W}$ and $1 \mathrm{~W}$ available. These developments will allow Ti:sapphire laser technology to be cost-effective for very high power, high performance ultrafast laser and amplifier systems. Further work to improve the overall performance and efficiency includes implementation of thin-disk type pumping of the Ti:sapphire medium [25] to allow for multipass pumping of a crystal with length comparable to the pump confocal parameter, and further investigation of possible carrier multiplication in blue pumped Ti:sapphire to minimize quantum defect, increase pump efficiency, and decreasing cooling requirements.

\section{Funding}

Department of Energy (DOE) (DE-SC-0009707). 